\title{
Hepatitis C infection at a tertiary hospital in South Africa: Clinical presentation, non-invasive assessment of liver fibrosis, and response to therapy
}

\author{
W B Abuelhassan, ${ }^{1}$ MBBS, MRCP, MRCP (Gastro), FCP (SA), MMed (Med), FRCP (Glasg); \\ G I Gasim, ${ }^{1,2}$ MBBS, MD (Med), MRCP (UK), ESEGH, FRCP (Glasg), Cert Gastroenterology (Phys) SA; \\ R Ally, ${ }^{1}$ MB ChB, FCP (SA), FRCP, MSc (Med); C Menezes, ${ }^{3}$ MD, MMed (Med), FCP (SA), DTM\&H, Cert ID (SA), PhD

\begin{abstract}
${ }^{1}$ Division of Gastroenterology, Chris Hani Baragwanath Academic Hospital and Faculty of Health Sciences, University of the Witwatersrand, Johannesburg, South Africa

${ }^{2}$ Faculty of Medicine, Alneelain University, Khartoum, Sudan

${ }^{3}$ Division of Infectious Diseases, Chris Hani Baragwanath Academic Hospital and Faculty of Health Sciences, University of the Witwatersrand, Johannesburg, South Africa
\end{abstract}

Corresponding author: W B Abuelhassan (wamdaabuelhassan@hotmail.com)

\begin{abstract}
Background. Hepatitis $\mathrm{C}$ is a viral infection that leads to chronic liver disease, resulting in significant morbidity and mortality. Objectives. To describe the demographic characteristics and clinical presentation of patients with chronic hepatitis $\mathrm{C}$ infection. The aspartate aminotransferase-to-platelet ratio index (APRI) and the fibrosis index based on 4 factors (FIB-4) were assessed for prediction of liver fibrosis. Methods. We retrospectively reviewed 87 records of patients who presented to the liver clinic at Chris Hani Baragwanath Academic Hospital, Johannesburg, South Africa, from January 2007 to December 2016. Patients' records were reviewed and analysed using SPSS statistical software version 24. Convenience sampling was used.

Results. The patients' mean (standard deviation (SD)) age was 52.6 (12.3) years. Fifty-four percent were female. Hepatitis $C$ virus genotype 5 was exclusively found in blacks $(p<0.001)$, constituting $60.3 \%$ of infections in this ethnic group and $48.7 \%$ in the cohort, followed by genotype 1 (21.8\%), genotype 3 (15.4\%), genotype 4 (10.3\%) and mixed-genotype infections (3.8\%). Genotype 5 patients were older (mean (SD) age 56.7 (9.8) years) than genotype 1 (46.3 (11.4) years) and genotype 3 (42 (9.8) years) ( $p=0.002$ and $p<0.001$, respectively). The receiver operating characteristic curve for METAVIR F0 v. APRI (cut-off $<0.7$ ) showed a moderate correlation, with an area under the curve (AUC) of 0.349 ( $p=0.002$ ), sensitivity of $78.8 \%$, specificity of $70.6 \%$ and a negative predictive value (NPV) of $63.2 \%$. METAVIR F4 v. APRI (cut-off $\geq 1.5$ ) showed an AUC of 0.881 ( $p=0.001$ ) with sensitivity of $85.7 \%$, specificity of $93 \%$ and a positive predictive value (PPV) of $67 \%$. METAVIR F0 v. FIB-4 (cut-off <1.45) showed a moderate correlation, with an AUC of 0.332 ( $p=0.021$ ), sensitivity of $78.3 \%$, specificity of $53.8 \%$ and an NPV of $73.7 \%$. METAVIR F4 v. FIB-4 (cut-off $>3.25$ ) had a strong correlation, with an AUC of $0.952(p<0.001)$, sensitivity of $63.6 \%$, specificity of $100 \%$ and a PPV of $100 \%$. Early virological response (EVR) was found to predict sustained virological response (SVR) to therapy (odds ratio $27.8 ; 95 \%$ confidence interval $2.8-274.3 ; p=0.004$ ).

Conclusions. Compared with other genotypes, genotype 5 was predominant in our cohort, particularly in older age groups. Moreover, APRI and FIB-4 scores correlated significantly with advanced fibrosis in HCV patients. Finally, EVR during therapy was found to determine SVR.
\end{abstract}

S Afr Med J 2020;110(9):920-925. https://doi.org/10.7196/SAMJ.2020.v110i9.14474

Hepatitis $\mathrm{C}$ is a viral infection that leads to chronic liver disease resulting in significant morbidity and mortality, with many patients progressing to liver cirrhosis and hepatocellular carcinoma. It tops the list of indications for liver transplantation in Japan, Europe and the USA. ${ }^{[1]}$ Seven major hepatitis $\mathrm{C}$ virus (HCV) genotypes have been recognised since the identification of HCV in 1989. These genotypes are one of the most critical factors determining response to therapy. ${ }^{[2]}$ Genotype 5 is reported to be the predominant HCV genotype in South Africa (SA), constituting $~ 40 \%$ of HCV infections, followed by genotype $1(33 \%){ }^{[3]}$

Risk factors for hepatitis C infection include blood and blood products transfusion and organ transplantation prior to 1992, intravenous drug use, long-term haemodialysis, multiple sexual partners, tattooing and scarification, unsafe medical practices, HIV infection, and being born to an HCV-infected mother. ${ }^{[4-7]}$ Interestingly, the ethnic background of patients has also been reported to determine the prevalence of HCV infection. A US study including $>5$ million individuals reported the highest prevalence of hepatitis $\mathrm{C}$ in blacks, followed by Hispanics. ${ }^{[8]}$

Anti-HCV antibodies are used for screening for hepatitis C. Thereafter qualitative and quantitative assays are used to detect the HCV virus RNA and determine the number of existing viral copies. Further tests are done to determine the genotypes and their subtypes.

Patients diagnosed with hepatitis $\mathrm{C}$ usually require a liver biopsy before starting treatment unless contraindicated, e.g. patients with thrombocytopenia and haemophilia. Liver biopsy is done to assess the degree of fibrosis and detect other liver disorders such as fatty liver and iron overload. According to the 2010 SA hepatitis C guidelines ${ }^{[9]}$ liver biopsy was not mandatory in $\mathrm{HCV}$ genotypes 2 and 3 because of excellent sustained viral response (SVR) rates (90\%) with the combination of pegylated interferon and ribavirin (PEG-RBV) in those individuals. ${ }^{[9]}$ The METAVIR score is usually used to report on the liver histology. ${ }^{[10]}$ Recently, non-invasive scores have been introduced as predictors of liver fibrosis. These include 
the aspartate aminotransferase-to-platelet ratio index (APRI) and the fibrosis index based on four factors (FIB-4). ${ }^{[1,12]}$ The APRI and FIB-4 scores have been reported to identify hepatitis C-related fibrosis in many studies, thus alleviating the need for the invasive liver biopsy. ${ }^{[10,13,14]}$ Sonderup et al. ${ }^{[15]}$ emphasised the importance of these scores in the assessment of liver fibrosis in the context of hepatitis $\mathrm{C}$ in sub-Saharan Africa, as they are cheap, available, easy to interpret and can be done in an outpatient setting. However, these scores have not been reported on in HCV-infected individuals in SA or sub-Saharan Africa.

Other useful non-invasive methods for evaluation of liver fibrosis/ cirrhosis include computed tomography liver surface nodularity scores, magnetic resonance elastography, and transient elastography (FibroScan) ${ }^{[16]}$ Lack of expertise and the high cost of these methods prevent their regular use. FibroScan uses measurement of liver stiffness to detect cirrhosis. While it is an excellent tool for assessment of liver cirrhosis, it is not readily available, especially in low-income settings. ${ }^{[17]}$

The standard treatment used for chronically infected patients with hepatitis C in SA was the combination of PEG-RBV for 24 or 48 weeks, with SVR achieved in up to $80 \%$ of those treated. ${ }^{[18]}$ However, achievement of an SVR is governed by the HCV genotype along with other factors such as genetic factors, e.g. interleukin (IL)-28B. ${ }^{[10,19]}$ Hadziyannis et al. ${ }^{[20]}$ showed that patients infected with genotype 1 need standard doses of ribavirin, while those infected with genotypes 2 and 3 are adequately treated with low doses. Given the serious side-effects of such a combination ${ }^{[21]}$ and obstacles to its use among large numbers of HCV patients, deciding whether to use the combination was not always easy. ${ }^{[22]}$ Since the introduction of directly acting antivirals (DAAs) in May 2011, hepatitis C treatment has evolved tremendously, especially with the introduction of pangenotypic regimens that conferred higher SVR rates with shorter duration of therapy.

Several factors are known to predict response to HCV therapy, including: (i) age, with age $<40$ years associated with a better response; (ii) race (whites do better than blacks in US studies); (iii) body mass index (BMI) (patients with a BMI $<25 \mathrm{~kg} / \mathrm{m}^{2}$ do better than those with higher BMIs); (iv) HCV genotype; $(v)$ viral load; ( $v i)$ rapid viral response; and (vii) the degree of liver fibrosis, and alpha-fetoprotein and $I L-28 B$ gene polymorphism. ${ }^{[23]}$

\section{Objectives}

Despite the impact of HCV infection on the SA population, especially in view of its frequent coexistence with the highly prevalent HIV infection, there is a lack of published data on HCV. This study therefore aimed to describe the demographic characteristics of patients with chronic hepatitis $\mathrm{C}$, disease presentation, clinical parameters, co-infection with hepatitis $\mathrm{B}$ and HIV, and response to therapy. It also aimed to assess the utility of the fibrosis score in detecting liver fibrosis. It is expected that the findings will help to provide data on the spectrum of hepatitis $\mathrm{C}$ infection in a referral hospital setting in SA and to shed light on the use of fibrosis scores in the SA setting.

\section{Methods}

The research protocol was approved by the Human Research Ethics Committee of the University of the Witwatersrand (ref. no. M170538).

\section{Study population}

We retrospectively reviewed 87 records of patients who presented to the liver clinic in the Division of Gastroenterology at Chris Hani Baragwanath Hospital (CHBAH), Johannesburg, between January 2007 and December 2016. No files were excluded. Patients diagnosed with hepatitis $C$ infection were referred from local clinics, the South African National Blood Service, CHBAH and private clinics. Their records were reviewed and analysed. Convenience sampling was used.

The datasets used and/or analysed during the study are available from the corresponding author (WBA) on reasonable request.

\section{Data collection}

A standardised data collection sheet was used. Data were extracted from records of all patients who attended the clinic. The results of baseline investigations, including a full blood count, liver function tests, urea and electrolytes, hepatitis C viral load and genotype, hepatitis B serology and HIV serology, were recorded. Liver biopsy results, where available, were extracted. A liver biopsy was performed in all consenting patients except where it was contraindicated or was not part of the treatment protocol (it was not done in patients with genotype 3). Patients eligible for treatment were treated with PEG-RBV. Hepatitis C viral load levels measured during treatment and at 24 weeks after treatment completion to determine SVR were extracted.

\section{Definitions}

Treated patients were deemed to have achieved a rapid virological response if they had an undetectable HCV viral load at week 4 of therapy. Early virological response (EVR) was defined as complete when viral load was undetectable at 12 weeks of treatment or as partial when there was a 2-log drop in viral load at 12 weeks of treatment. SVR was defined as an undetectable HCV viral load 24 weeks after completion of treatment.

\section{Statistical analysis}

All data were analysed using SPSS statistical software, version 24.0 for Windows (SPSS Inc., USA). Categorical data such as gender, ethnicity and symptoms were analysed using Pearson's $\chi^{2}$ test or Fisher's exact test when $>20 \%$ of cells had an expected frequency $<5$. Continuous data such as age and viral load were analysed using parametric methods and presented as means and standard deviations (SDs) or medians and percentiles for non-normally distributed data. The geometric mean was used to analyse BMI and viral load. The $t$-test was used in the analysis of normally distributed continuous data, and one-way analysis of variance with Dunett's correction was used to compare the ages of patients with the different genotypes; otherwise the Mann-Whitney $U$-test was used. Multinomial logistic regression was used to determine the risk factors for HCV infection in the study cohort after calculating their univariate logistic regression. The Wilcoxon signed-rank test was used for analysis of treatment effect on viral load and liver enzymes. Eta correlation was used to assess the correlation between the METAVIR, APRI and FIB- 4 scores, while Spearman's correlation was used for the correlation between APRI and FIB-4 scores. The receiver operating characteristic (ROC) curve was also used to compare APRI and FIB- 4 with the gold standard METAVIR. A $p$-value of $<0.05$ was considered statistically significant.

\section{Results}

\section{Demographics}

Eighty-seven patients' records were identified (Fig. 1). The mean (SD) age of the cohort at presentation was 52.6 (12.3) years. Female patients constituted $53.5 \%$ of the group. There were 71 blacks (81.7\%), 9 Asians (10.3\%) and 7 whites (8.0\%). Black HCV-infected patients tended to be older compared with other ethnic groups, with a mean (SD) age of 55.7 (10.6) years, which was statistically significant $(p=0.001)$ (Table 1$)$. 


\section{Risk factors}

Twenty-three of the black patients had a history of blood transfusion, but this was not statistically significant. Five individuals had haemophilia. There was no statistically significant difference in the prevalence of haemophilia between the different ethnic groups. White patients had more tattooing and history of intravenous drug use than blacks and Asians, which was statistically significant $(p<0.001)$ (Table 1). Histories of previous surgery and scarification were not significantly different between the ethnic groups. Data for men who have sex with men were not available.

\section{Clinical presentation}

Thirty-one patients (35.6\%) were incidentally diagnosed. Forty-seven (60.3\%) were symptomatic. In 9 patients (10.3\%), presentation was

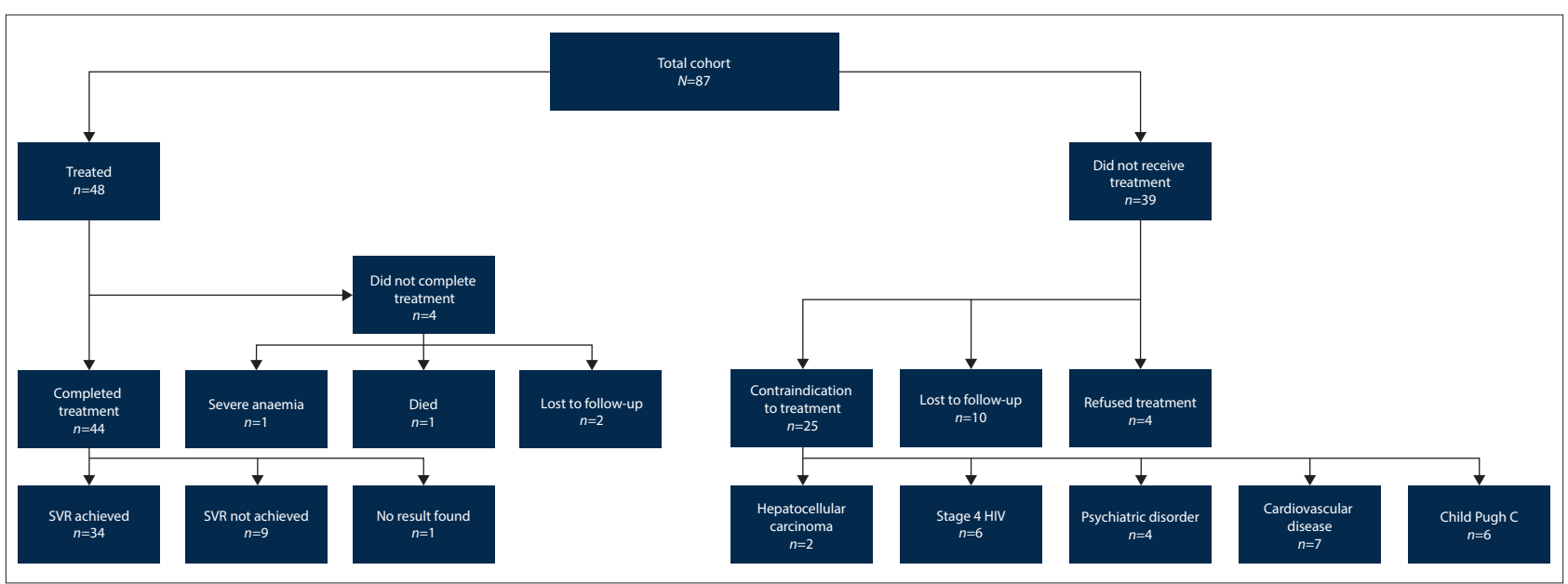

Fig. 1. Flow chart of the study cohort. (SVR= sustained virological response.)

Table 1. Hepatitis C infection: Cohort characteristics according to ethnicity

\begin{tabular}{|c|c|c|c|}
\hline \multirow[b]{2}{*}{ Characteristics } & \multicolumn{3}{|c|}{ Ethnicity } \\
\hline & Black $(N=71)$ & White $(N=7)$ & Asian $(N=9)$ \\
\hline Age (years), mean (SD) & $55.7(10.6)$ & $33.1(3.9)$ & $43.6(11.6)$ \\
\hline \multicolumn{4}{|l|}{ Gender, $n(\%)$} \\
\hline Male & $33(46.5)$ & $3(42.9)$ & $4(44.4)$ \\
\hline Female & $38(53.5)$ & $4(57.1)$ & $5(55.6)$ \\
\hline \multicolumn{4}{|l|}{ Comorbidities, $n(\%)$} \\
\hline $\mathrm{DM}$ & $8(11.3)$ & 0 & $2(22.2)$ \\
\hline CKD & $8(11.3)$ & 0 & 0 \\
\hline Haemophilia & $4(5.65)$ & $1(14.3)$ & 0 \\
\hline Chronic hepatitis B & $2(2.83)$ & 0 & $1(11.1)$ \\
\hline HIV & $16(22.6)$ & $1(14.3)$ & 0 \\
\hline BMI, mean (SD) & $25.1(1.2)$ & $24(1.2)$ & $26.3(1.15)$ \\
\hline \multicolumn{4}{|l|}{ Risk factors, $n(\%)$} \\
\hline Blood transfusion & $23(32.4)$ & $1(14.3)$ & $2(22.2)$ \\
\hline Tattoo & 0 & $3(42.9)$ & 0 \\
\hline Scarification & $1(1.4)$ & 0 & 0 \\
\hline IV drug use & 0 & $3(42.9)$ & 0 \\
\hline Surgery & $11(15.5)$ & 0 & $2(22.2)$ \\
\hline GTs $(N=78), n(\%)$ & $63(80.8)$ & $6(7.7)$ & $9(11.5)$ \\
\hline GT 1 & $11(17.5)$ & $2(33.3)$ & $4(44.4)$ \\
\hline GT 3 & $3(4.8)$ & $4(66.6)$ & $5(55.6)$ \\
\hline GT 4 & $8(12.7)$ & 0 & 0 \\
\hline GT 5 & $38(60.3)$ & 0 & 0 \\
\hline$>1 \mathrm{GT}$ & $3(4.8)$ & 0 & 0 \\
\hline $\mathrm{VL}$, mean $(\mathrm{SD})$ & $\log 5.8(0.8)$ & $\log 5.6(1.2)$ & $\log 5.5(1.2)$ \\
\hline Total treated $(N=48), n(\%)$ & $37(52.1)$ & $5(71.4)$ & $6(66.7)$ \\
\hline Response/non-response to treatment, $N$ & 34 & 5 & 5 \\
\hline SVR, $n(\%)$ & $27(79.4)$ & $5(100)$ & $2(40.0)$ \\
\hline Non-responders, $n(\%)$ & $5(14.7)$ & 0 & $2(40.0)$ \\
\hline Relapse, $n(\%)$ & $2(5.9)$ & 0 & $1(20.0)$ \\
\hline
\end{tabular}


not documented. Of symptoms and signs, the most frequent was joint pain $(n=11$; $12.8 \%)$. This was followed in frequency by ascites $(n=6 ; 6.9 \%)$, lower limb oedema $(n=5 ; 5.7 \%)$ and jaundice $(n=5 ; 5.7 \%)$. Of the 78 patients for whom the information was available, 9 were smokers; $13 / 81$ patients consumed alcohol. The geometric mean (SD) BMI was 25.12 (1.2).

\section{Comorbidities}

Comorbidities in this cohort included diabetes mellitus ( $n=10$ patients), chronic kidney disease ( $n=8$ black patients) and cardiovascular disease $(n=7)$. There was no statistically significant difference in the prevalence of comorbidities between the different ethnic groups. Of the 87 patients, 3 (2 blacks and 1 Asian) had chronic hepatitis B, while 17 patients had HIV infection $(n=16(22.5 \%)$ black and $n=1(14.3 \%)$ white patients). The median (interquartile range) CD4+ count for the HIV co-infected patients was $448(283-900)$ cells $/ \mu \mathrm{L}$.

\section{Genotypes}

Genotypes were available for 78 patients. Genotype 5 was exclusively found in blacks. It constituted $60.3 \%$ of infections in this ethnic group and $48.7 \%$ in the cohort, followed by genotype $1(21.8 \%)$, genotype 3 (15.4\%), genotype $4(10.3 \%)$ and mixedgenotype infection (3.8\%). When genotype 5 was compared with HCV genotypes in other ethnic groups, a statistically significant difference was evident $(p<0.001)$. Genotype 5 patients were found to be older, with a mean (SD) age of 56.7 (9.8) years, compared with genotype 1 (46.3 (11.4) years) and genotype 3 (42 (9.8) years) $(p=0.002$ and $p<0.001$, respectively).

\section{Viral loads}

The mean (SD) viral load for the cohort was $\log 5.7(0.9)$. There was no statistical difference between the different ethnic groups or different genotypes in terms of viral load levels.

\section{METAVIR and fibrosis scores}

Fifty-nine patients, of whom 10 were known to be HIV-positive, had liver biopsy results. The mean (SD) platelet count for patients with HIV was $297(92) \times 10^{9} / \mathrm{L}$. Metavir and APRI scores and FIB-4 and APRI scores were found to be strongly correlated ( $r=1$, 0.997 and 0.817 , respectively). The ROC curve for METAVIR F0 and APRI (cut-off $<0.7)$ showed a moderate correlation, with an AUC of $0.349(p=0.002)$, sensitivity of $78.8 \%$, specificity of $70.6 \%$ and a negative predictive value (NPV) of $63.2 \%$, and that for METAVIR F4 v. APRI (cut-off $\geq 1.5$ ) had an AUC of $0.881(p=0.001)$ with sensitivity of $85.7 \%$, specificity of $93 \%$ and a positive predictive value (PPV) of $67 \%$ (Fig. 2). METAVIR F0 v. FIB-4 (cut-off <1.45) had a moderate correlation, with an AUC of 0.332 $(p=0.021)$, sensitivity of $78.3 \%$, specificity of $53.8 \%$ and an NPV of $73.7 \%$. METAVIR F4 v. FIB-4 (cut-off $>3.25$ ) had a strong correlation, with an AUC of $0.952(p<0.001)$, sensitivity of $63.6 \%$, specificity of $100 \%$ and a PPV of $100 \%$ (Fig. 3).

\section{Treatment}

All treated patients received pegylated interferon $\alpha-2 a$ (Pegasys) and ribavirin. Of 48 patients who were treated, 44 completed treatment; 34 patients achieved SVR while 9 did not, and in 1 case results were not available (Fig. 1). All but 1 of the patients developed side-effects secondary to treat- ment. Anaemia was the most common side-effect $(n=33 ; 68.8 \%)$, followed by leukopenia $(n=31 ; 65 \%)$ and fatigue $(n=27$; $56.2 \%$ ). Growth factors (erythropoietin and granulocyte-colony stimulating factor) were used in $17(35.4 \%)$ and $6(12.5 \%)$ patients, respectively, during treatment.

Both alanine aminotransferase and aspartate aminotransferase showed a statistically significant decline post treatment $(p<0.001)$. EVR was found to predict SVR to therapy (odds ratio 27.8; 95\% confidence interval 2.8 - 274.3; $p=0.004$ ) (Table 2).

\section{Discussion}

Chronic HCV infection causes significant morbidity and mortality, as it remains asymptomatic for prolonged periods of time until the individual develops chronic liver disease and/or HCC, yet it is potentially curable. ${ }^{[2]}$

As HCV has the same mode of transmission as HIV, and the prevalence of HIV in SA is high, SA would be expected to have a large burden of patients with $\mathrm{HCV}$ infection. There is a paucity of data on $\mathrm{HCV}$ infection in SA. We describe the clinical characteristics of our cohort of 87 patients, with specific emphasis on genotype 5 . This genotype is endemic in SA, but there is a paucity of literature on it worldwide. ${ }^{[24]}$ Genotype 5 was seen in $48.7 \%$ of the patients in our cohort; this is similar to what Smuts et al. ${ }^{[3]}$ described in a sample of 79 patients. On the other hand, Prabdial-Sing et al. ${ }^{[24]}$ described a slightly lower proportion of genotype 5 infections (36\%) in a larger cohort studied at the National Health Laboratory Service. Ethnic groups were not identified in their study. In the present study, genotype 5 was found exclusively in SA

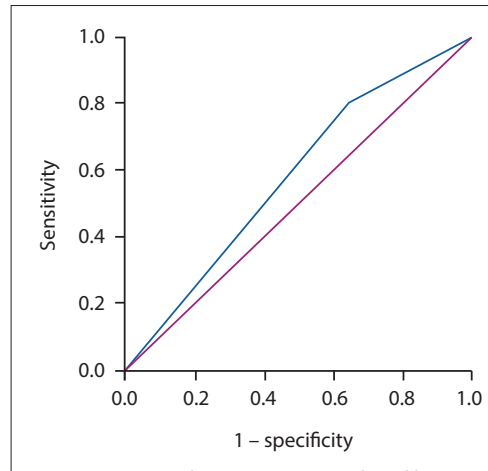

Diagonal segments are produced by ties

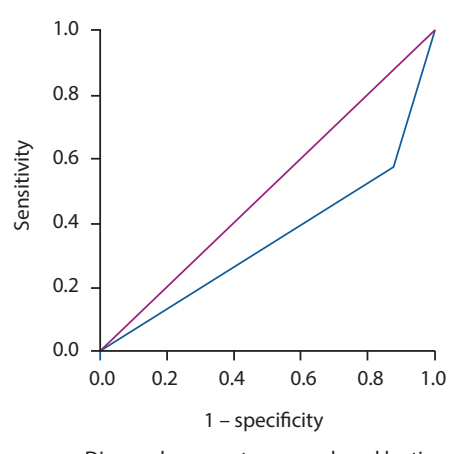

Diagonal segments are produced by ties



Diagonal segments are produced by ties

5) V. METAVIR F4

AUC $0.578, p=0.571$

Sensitivity $20 \%$

Specificity $6 \%$

PPV $79 \%$

AUC $0.881, p<0.001$

Sensitivity $85.7 \%$

Specificity $93 \%$

PPV $67 \%$

Fig. 2. ROC curve showing correlation between APRI and METAVIR scores. (ROC = receiver operating characteristic; APRI = aspartate aminotransferaseto-platelet ratio index; $A U C=$ area under the curve; $N P V=$ negative predictive value; $P P V=$ positive predictive value.) 


\begin{tabular}{|c|c|c|c|}
\hline Variables & SVR & No SVR & $p$-value \\
\hline Age (years), mean (SD) & $47.2(11.8)$ & $54.7(9.9)$ & 0.103 \\
\hline Gender, $n$ & & & 0.889 \\
\hline Male $(N=19)$ & 15 & 4 & \\
\hline Female $(N=24)$ & 18 & 6 & \\
\hline Ethnicity, $n$ & & & 0.090 \\
\hline White $(N=5)$ & 5 & 0 & \\
\hline Black $(N=34)$ & 27 & 7 & \\
\hline Asian $(N=4)$ & 2 & 2 & \\
\hline $\operatorname{HIV}(N=4), n$ & 4 & 0 & 0.999 \\
\hline GTs, $n$ & & & 0.079 \\
\hline GT 1 & 5 & 3 & \\
\hline GT3 & 5 & 1 & \\
\hline GT4 & 1 & 3 & \\
\hline GT5 & 20 & 2 & \\
\hline$>1 \mathrm{GT}$ & 3 & 0 & \\
\hline $\operatorname{RVR}(N=27), n$ & 23 & 4 & 0.211 \\
\hline EVR week $12(N=43), n$ & 34 & 9 & $0.004^{*}$ \\
\hline FIB-4 $(N=40), n$ & 33 & 7 & 0.036 \\
\hline APRI $(N=40), n$ & 33 & 7 & 0.079 \\
\hline
\end{tabular}

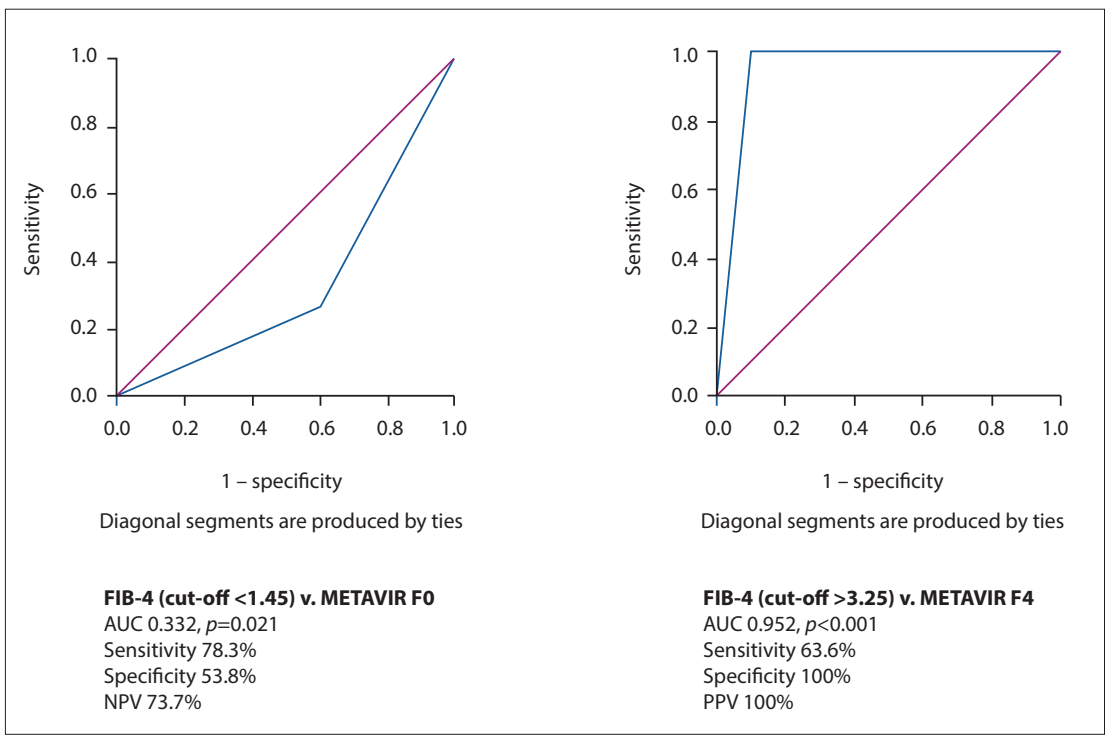

Fig. 3. ROC curve showing correlation between FIB- 4 and METAVIR scores. (ROC $=$ receiver operating characteristic; $F I B-4=$ fibrosis index based on 4 factors; $A U C=$ area under the curve; NPV = negative predictive value; $P P V=$ positive predictive value.)

blacks. This could possibly be attributed to segregation of racial groups under the previous apartheid regime, particularly as patients infected with genotype 5 were found to be significantly older than those with genotypes 1 and 3. The observation that genotype 5-infected patients were older was also made by French researchers in $2004 .{ }^{[25]}$ Genotypes 1,3 and 4 and mixedgenotype infections followed genotype 5 in frequency, with percentages of $21.8 \%$, $15.4 \%, \quad 10.3 \%$ and $3.8 \%$, respectively, a distribution similar to that described by Prabdial-Sing et al., ${ }^{[24]}$ who reported prevalences of $22 \%$ for genotype $1 \mathrm{~b}, 11.7 \%$ for genotype $3 \mathrm{a}$ and $8.91 \%$ for genotype 4 , while the prevalence of mixed infections was $7 \%$. On the other hand, these figures are much higher than those reported by Smuts et al. ${ }^{[3]}$ although the sample sizes were not dissimilar.

Rates of hepatitis B infection were low in our cohort, despite the shared modes of transmission of those infections with HCV while slightly more than a fifth of patients had HIV co-infection.
Many guidelines on hepatitis $\mathrm{C}$ treatment recommend assessment of liver fibrosis/ cirrhosis before initiation of therapy. ${ }^{[9,15]}$ While liver biopsy has been deemed the gold standard, implementing it in all settings can be difficult owing to high cost, lack of expertise and cultural unacceptability. ${ }^{[15]}$ Several non-invasive methods of liver fibrosis assessment have been studied and developed. These include the APRI and FIB4, which have not been studied previously in our setting. In our cohort, scores for liver fibrosis such as the APRI and FIB-4 correlated significantly with an advanced METAVIR score of fibrosis (METAVIR F3 and F4). Similar results have been reported by others. ${ }^{[13,14]}$ Implementing these scores in clinical practice can reduce the need for invasive liver biopsies. Interestingly, the same fibrosis scores have been found to have limitations when used in hepatitis $B$ patients. Conventional cut-offs for these scores cannot be used to detect cirrhosis in the setting of hepatitis $\mathrm{B}$, owing to high misclassification rates. ${ }^{[2,27]}$

Various factors have been found to affect clearance of the virus, but in our cohort week 12 EVR was the only predictor of SVR. Rao et al. ${ }^{[18]}$ came to a similar conclusion in their multicentre study that included 125 chronic hepatitis $\mathrm{C}$ patients.

\section{Study strengths and limitations}

The major strength of this study is that it adds significant information on the clinical characteristics of patients with HCV in SA, 
with assessment of the utility of fibrosis scores, even though it was a retrospective single-centre study and did not include patients who had been treated with DAAs. However, this situation has since changed, and DAAs are going to be the standard of care for $\mathrm{HCV}$ patients in SA.

\section{Conclusions}

The current study concludes that genotype 5 is the predominant genotype in a single SA centre. It is more common than other genotypes among older SA blacks. Moreover, the APRI and FIB-4 had sufficient power to diagnose advanced fibrosis in HCV patients, alleviating the need for liver biopsy in selected individuals. Despite their shared modes of transmission, hepatitis B and HIV infections were not commonly encountered in HCV patients in this cohort. Finally, EVR was found to determine who is likely to have an SVR.

Declaration. The research for this study was done in partial fulfilment of the requirements for WBA's MMed (Med) degree at the University of the Witwatersrand.

\section{Acknowledgements. None.}

Author contributions. WBA wrote the manuscript. GIG analysed the patient data and assisted in writing the manuscript. RA and CM assisted in writing the manuscript. All authors read and approved the final manuscript.

Funding. Privately funded.

Conflicts of interest. None.

1. Oliver M, Ortiz CC, Ortiz J. Challenging hepatitis C-infected liver transplant patients. Hepat Med 2016;8:1-8. https://doi.org/10.2147/HMER.S96110

2. European Association for the Study of the Liver. EASL Recommendations on Treatment of Hepatitis C 2015. J Hepatol 2015;63(1):199-236. https://doi.org/10.1016/j.jhep.2015.03.025

3. Smuts HE, Kannemeyer J. Genotyping of hepatitis C virus in South Africa. J Clin Microbiol 1995;33(6):1679-1681.

4. Gasim GI, Murad IA, Adam I. Hepatitis B and C virus infections among pregnant women in Arab and African countries. J Infect Dev Ctries 2013;7(8):566-578. https://doi.org/10.3855/jidc.3243

5. Merican I, Sherlock S, McIntyre N, Dusheiko GM. Clinical, biochemical and histological features in 102 patients with chronic hepatitis C virus infection. Q J Med 1993;86(2):119-125.

6. Gasim GI, Hamdan HZ, Hamdan SZ, Adam I. Epidemiology of hepatitis B and hepatitis C virus infections among hemodialysis patients in Khartoum, Sudan. J Med Virol 2012;84(1):52-55. https:// doi.org/10.1002/imv.22256

7. Murad EA, Babiker SM, Gasim GI, Rayis DA, Adam I. Epidemiology of hepatitis B and hepatitis C Murad EA, Babiker SM, Gasim GI, Rayis DA, Adam I. Epidemiology of hepatitis B and hepatitis C
virus infections in pregnant women in Sanaa, Yemen. BMC Pregnancy Childbirth 2013;13(1):127. virus infections in pregnant women in S
https://doi.org/10.1186/1471-2393-13-127
8. Backus LI, Belperio PS, Loomis TP, Mole LA. Impact of race/ethnicity and gender on HCV screening and prevalence among US veterans in Department of Veterans Affairs care. Am J Public Health 2014;104(Suppl 4):555-561. https://doi.org/10.2105/AJPH.2014.302090

9. Botha JF, Kassianides C, Schneider HR, Song E, Spearman W, van der Merwe SW. South African Hepatitis C Management Guidelines 2010. S Afr Gastroenterol Rev 2010;8(1):20-25. http://www. Hepatitis C Management Guidelines 2010. S Afr Gastroenterol Rev 2010;8(1):20-25. http://
sages.co.za/content/images/SouthAfricanHepatitisCGuidelines.pdf (accessed 23 March 2019).

10. Bages.co.za/content/images/SouthAfricanHepatitisC Guidelines.pdf (accessed 23 March 2019).
10, Poynard T. An algorithm for the grading of activity in chronic hepatitis C: The METAVIR Cooperative Study Group. Hepatology 1996;24(2):289-293. https://doi.org/10.1002/hep.510240201

11. Lin ZH, Xin YN, Dong QJ, et al. Performance of the aspartate aminotransferase-to-platelet ratio index for the staging of hepatitis C-related fibrosis: An updated meta-analysis. Hepatology 2011;53(3):726-736. https://doi.org/10.1002/hep.24105

12. Sterling RK, Lissen E, Clumeck N, et al. Development of a simple noninvasive index to predict significant fibrosis in patients with HIV/HCV coinfection. Hepatology 2006;43(6):1317-1325. https://doi.org/10.1002/hep.21178

13. De Oliveira AC, El-Bacha I, Vianna MV, Parise ER. Utility and limitations of APRI and FIB4 to predict staging in a cohort of nonselected outpatients with hepatitis C. Ann Hepatol 2016;15(3):326332. https://doi.org/10.5604/16652681.1198801

14. Amorim TGF, Staub GJ, Lazzarotto C, et al. Validation and comparison of simple noninvasive Amorim TGF, Staub G), Lazzarotto C, et al. Validation and comparison of simple noninvasive
models for the prediction of liver fibrosis in chronic hepatitis C. Ann Hepatol 2012;11(6):855-861. https://doi.org/10.1016/S1665-2681(19)31410-3

15. Sonderup MW, Afihene M, Ally R, et al. Hepatitis C in sub-Saharan Africa: The current status and recommendations for achieving elimination by 2030. Lancet Gastroenterol Hepatol 2017;2(12):910919. https://doi.org/10.1016/S2468-1253(17)30249-2

16. Smith AD, Porter KK, Elkassem AA, Sanyal R, Lockhart ME. Current imaging techniques for noninvasive staging of hepatic fibrosis. AJR Am J Roentgenol 2019;213(1):1-13. https://doi. org/10.2214/AJR.19.21144

17. Foucher J, Chanteloup E, Vergniol J, et al. Diagnosis of cirrhosis by transient elastography (FibroScan): A prospective study. Gut 2006;55(3):403-408. https://doi.org/10.1136/gut.2005.069153

18. Rao H, Yang R, Shang J, et al. [The efficacy and prognostic predictors of different treatment courses with pegylated interferon alpha-2a and ribavirin combination in recurrent chronic hepatitis C patients]. Zhonghua Nei Ke Za Zhi 2015;54(8):699-704. https://doi.org/10.3760/cma.j. issn. 0578-1426.2015.08 .011

19. Mangia A, Santoro R, Sarli R, et al. IL28B CC-genotype association with HLA-DQB1*0301 allele increases the prediction of spontaneous HCV RNA clearance in thalassaemic HCV-infected increases the prediction of spontaneous HCV RNA clearance in thalassa

20. Hadziyannis SJ, Sette H, Morgan TR, et al. Peginterferon-alpha2a and ribavirin combination therapy in chronic hepatitis C: A randomized study of treatment duration and ribavirin dose. Ann Intern in chronic hepatitis C: A randomized study of treatment duration and ribavirin dose.
Med 2004;140(5):346-355. https://doi.org/10.7326/0003-4819-140-5-200403020-00010

21. Mahmood K, Muhammad N. Side effects of combination of interferon plus ribavirin therapy in patients with chronic hepatitis C: An experience with 400 patients. J Postgrad Med Inst 2007;21(3):187-191.

22. Ghany MG, Strader DB, Thomas DL, Seeff LB. Diagnosis, management, and treatment of hepatitis C: An update. Hepatology 2009;49(4):1335-1374. https://doi.org/10.1002/hep.22759

23. Davis GL, Lau JY. Factors predictive of a beneficial response to therapy of hepatitis C. Hepatology 1997;26(Suppl 1):122S-127S. https://doi.org/10.1002/hep.510260721

24. Prabdial-Sing N, Puren A, Schoub B. The status of hepatitis C - the silent 'volcano' - in South Africa. Natl Inst Commun Dis Commun Dis Surveill Bull 2013;11(1):22-25.

25. Henquell C, Cartau C, Abergel A, et al. High prevalence of hepatitis $C$ virus type 5 in central France evidenced by a prospective study from 1996 to 2002. J Clin Microbiol 2004;42(7):3030-3035. https:// doi.org/10.1128/JCM.42.7.3030-3035.2004

26. Sonneveld MI, Brouwer WP, Chan HL. Optimisation of the use of APRI and FIB-4 to rule out cirrhosis in patients with chronic hepatitis B: Results from the SONIC-B study. Lancet Gastroenterol Hepatol 2019;4(7):538-544. https://doi.org/10.1016/S2468-1253(19)30087-1

27. Cooke GS, Andrieux-Meyer I, Applegate T, et al. Accelerating the elimination of viral hepatitis: A Lancet Gastroenterology \& Hepatology Commission. Lancet Gastroenterol Hepatol 2019;4(2):135184. https://doi.org/10.1016/S2468-1253(18)30270-X

Accepted 3 March 2020 